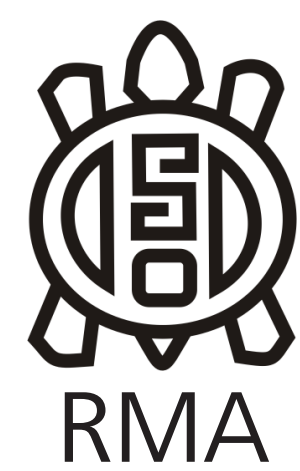

Arqueología

\title{
Acumulación de restos de lagartijas en el sitio arqueológico Orejas de Burro 1 (Provincia de Santa Cruz, Argentina)
}

\author{
Accumulation of lizard remains in the archaeological site Orejas de \\ Burro 1 (province of Santa Cruz, Argentina) \\ Adriana Albino
}

Departamento de Biología, Universidad Nacional de Mar del Plata-CONICET, Funes 3250, B7602AYJ Mar del Plata, Argentina; Email: aalbino@mdp.edu.ar

\begin{abstract}
Resumen
En la presente contribución se da a conocer una acumulación de restos de lagartijas recuperados en el sitio arqueológico Orejas de Burro 1 (OB1), ubicado en el campo volcánico Pali Aike, sur de la provincia de Santa Cruz (Argentina). Los materiales incluyen restos de coronoides, dentarios, frontales, maxilares, huesos largos, parietales, premaxilares, vértebras y fragmentos de otros huesos. Las características osteológicas permiten la asignación taxonómica de estos elementos al género Liolaemus. Hasta el momento se ha contabilizado un número superior a 100 dentarios izquierdos y 100 dentarios derechos, que permiten sostener la presencia de al menos 100 individuos. La abundancia de bolos de regurgitación de lechuzas hallados en el sitio explicaría el ingreso de los restos de lagartijas. El hallazgo en OB1 es el tercer reconocimiento del género Liolaemus en un sitio arqueológico de Argentina, constituye el hallazgo más austral conocido, y se destaca por la gran abundancia y diversidad de elementos preservados. Hasta el presente, los sitios arqueológicos de Patagonia se caracterizan por la homogeneidad taxonómica de lagartijas, donde el único taxón presente es Liolaemus.
\end{abstract}

Palabras clave: Iguania; zooarqueología; Liolaemus; Patagonia

\begin{abstract}
In the present contribution lizards remains recovered in the archaeological site Orejas de Burro 1 (OB1), located in the volcanic field PaliAike, South of the province of Santa Cruz (Argentina) are described. Materials include remains of coronoids, dentaries, frontals, maxillae, premaxillae, long bones, parietals, vertebrae and other bone fragments. Osteological features allow the taxonomic assignment of these elements to the genus Liolaemus. Until now, more than 100 left dentaries and 100 right dentaries have been recognized, supporting the presence of at least 100 individuals. The abundance of owl regurgitation pellets would explain the entrance of the lizard remains in the site. The record of lizards in OB1 is the third recognition of the genus Liolaemus in an archaeological site of Argentina, constituting the southernmost finding until now, and the most abundant and diverse in osteological elements. So far, the archaeological sites of Patagonia are characterized by the taxonomic homogeneity of lizards, where the only taxon recognized is Liolaemus.
\end{abstract}

Key words: Iguania; zooarchaeology; Liolaemus; Patagonia

Los estudios arqueofaunísticos en Argentina han enfatizado tradicionalmente el análisis de los restos de mamíferos, quedando relegados otros vertebrados que son conspicuos integrantes de las comunidades faunísticas en la actualidad, como los reptiles escamosos (lagartos, anfisbenas y serpientes), uno de los grupos que menor atención ha suscitado desde la zooarqueología. A pesar de ésto, y a partir de hallazgos realizados durante los últimos años, se ha podido documentar la importancia que tienen los escamosos en el análisis de las estrategias de explotación de recursos, en la interpretación del origen tafonómico de restos de pequeños vertebrados y en la valoración de la microfauna en los sistemas de creencias indígenas (Quintana y Mazzanti 2001; Albino y Albino 2004; Albino y Kligmann 2007, 2009; Kligmann et al. 2010; Albino y Franco 2011). De esta manera, ante el hallazgo de especímenes de escamosos en un sitio arqueológico, se evidencia la necesidad de realizar su determinación taxonómica al más bajo nivel sistemático posible y con sólida fundamentación para poder elaborar interpretaciones precisas.

Los lagartos iguánidos que habitan en la Argentina son conocidos mayormente con el nombre común de "lagartijas", sin embargo, incluyen una gran variedad de géneros y especies, algunos de los cuales viven en los más 
inhóspitos ambientes del extremo sur del continente y en las alturas de los Andes. Aunque se ha documentado la presencia de iguánidos en varios sitios arqueológicos de Argentina, sólo fueron formalmente descriptos los restos procedentes de sitios de Mendoza, Catamarca y Santa Cruz (Van Devender 1977; Albino y Kligmann 2007; Albino y Franco 2011). A estos hallazgos se agrega en este trabajo una notable acumulación de restos de lagartijas que se hallaban en el interior de una cueva (OB1), ubicada en el cráter del volcán Orejas de Burro, en el extremo meridional de la provincia de Santa Cruz.

\section{Materiales y métodos}

El volcán Orejas de Burro se localiza en el campo volcánico Pali Aike, sur de la provincia de Santa Cruz, Argentina. El sitio OB1 se ubica en el sector sureste del campo de lava ( $52^{\circ} 07.769^{\prime} \mathrm{S}, 69^{\circ} 33.151^{\prime} \mathrm{O}$ ), en una cueva de $12 \times 5 \mathrm{~m}$ ubicada en la ladera interna del cono volcánico (Barberena et al. 2006). El conjunto faunístico recuperado en el sitio está compuesto por más de 21.600 restos óseos, dentro de los cuales el taxón mejor representado es el de los roedores, con más de 16.000 huesos asignables a Ctenomys sp. (L'Heureux 2008). Los especímenes de lagartijas corresponden predominantemente a momentos posteriores al Holoceno tardío final (ca. 1.700-500 años ${ }^{14} \mathrm{C}$ AP, L'Heureux 2008). El mapa de la ubicación del sitio OB1, así como los detalles geoarqueólogicos y arqueofaunísticos se encuentran en Barberena et al. (2006), L'Heureux (2008) y L'Heureux y Barberena (2008).

La sistemática de los lagartos Iguania que se sigue en este trabajo es la propuesta por Schulte et al. (2003) que acepta la monofilia de los Iguanidae, dentro de los cuales se incluyen ocho subfamilias, distribuidas principalmente del Nuevo Mundo.En particular, la monofilia de la subfamilia Tropidurinae* no ha sido probada, por lo cual se la considera un metataxón, y en consecuencia, se denota con un asterisco.

Para la determinación de los restos de lagartijas se consideraron caracteres provistos por análisis filogenéticos (Frost y Etheridge 1989; Etheridge 1995, 2000; Frost et al. 2001; Lobo 2001) y confrontación directa con material osteológico comparativo de especies actuales depositado en la Colección Herpetológica de la Universidad Nacional de Mar del Plata - Sección Osteología (UNMdP-O).

Los restos se hallaron completamente desarticulados $y$, en la mayoría de los casos, son sólo fragmentos de huesos, algunos de los cuales están muy deteriorados y presentan una gran fragilidad. Se han preservado restos de coronoides, dentarios, frontales, maxilares, huesos largos, parietales, premaxilares, vértebras y fragmentos mal preservados de otros huesos. La cuantificación de partes esqueletarias según la categoría y distribución espacial se encuentra en proceso. Hasta el momento se ha contabilizado un número superior a 100 dentarios izquierdos y 100 dentarios derechos, que permiten sostener la presencia de un mínimo de 100 individuos.

\section{Resultados}

La posición sistemática de los restos de lagartijas recuperados en la muestra es la siguiente:

Squamata Oppel, 1811

Iguania Cope, 1864

Iguanidae Bell, 1825

Tropidurinae* Schulte et al., 2003

Género Liolaemus Wiegmann, 1834 Liolaemus sp.

Los caracteres que permiten la identificación de los materiales son: frontales fusionados y con fuerte constricción entre las órbitas; parietales fusionados y de forma trapezoidal; dientes de maxilares y dentarios tricuspidados, no fusionados al hueso, de implantación pleurodonte;zonas de resorción dentaria basal y lingual; dentarios con la cicatriz del proceso anterolateral del coronoides en forma de un ala bien desarrollada; dentarios con canal de Meckel abierto; alcance anterior del esplenial mayor a 1/6 y menor a la mitad de longitud de la serie dentaria; alcance posterior de dentarios superando el nivel del eje del proceso dorsal del coronoides; dientes posteriores de dentarios y maxilares cilíndricos, con coronas tricuspidadas, donde la cúspide central es mayor que las laterales, las cuales están bien desarrolladas y definidas (Figura 1).

De las casi 50 especies de lagartos que habitan la Patagonia sur, la mayoría corresponde al género Liolaemus (Scolaro 2005). La presencia de canal de Meckel abierto en los dentarios excluye a los restos de OB1 del grupo chiliensis, donde dicho canal está cerrado (Etheridge 1995). Dentro

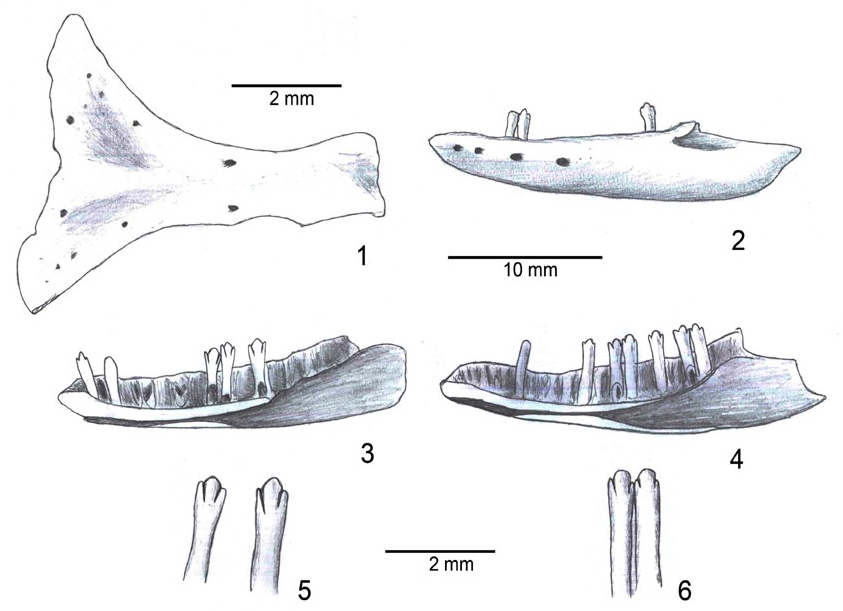

Figura 1: Algunos de los huesos de Liolaemus sp. recuperados en el sitio OB1. Frontal en vista dorsal (1), dentario en vista labial (2), dentarios en vista lingual $(3,4)$, detalles de los dientes $(5,6)$.

Figure 1: Some of the bones of Liolaemus sp. recovered in the site OB1. Frontal in dorsal view (1), dentary in labial view (2), dentaries in lingual view $(3,4)$, details of the teeth $(5,6)$. 
de las especies restantes, se descarta la pertenencia al grupo montanus, que se caracteriza por poseer la tibia con un proceso en forma de navaja (Etheridge 1995), ausente en las tibias preservadas en OB1. La osteología no permite discriminar entre la mayoría de las restantes especies, de manera que el material es referido como Liolaemus sp.; sin embargo, las especies que comparten los mismos caracteres y alcanzan las más altas latitudes en el extremo meridional de la provincia son $L$. kingii, $L$. lineomaculatus, L. magellanicus y L. sarmientoi de las cuales sólo las dos últimas se encuentran en el área del volcán Orejas de Burro (Scolaro 2005; Breitman et al. 2014). A través de la comparación directa, surge que el tamaño de los ejemplares más grandes de OB1 es únicamente compatible con las dimensiones de L. kingii y L. sarmientoi, de manera que probablemente se trate de restos de esta última especie debido a que $L$. kingii no supera la latitud de Puerto Santa Cruz (Scolaro 2005). La heterogeneidad en tamaños representados en el sitio impide descartar la presencia también de otra especie más pequeña. Además, algunos dentarios de dimensiones comparables tienen dientes ligeramente diferentes, mientras unos son más bajos y robustos, con cúspides muy profundamente diferenciadas y divergentes (Figura 1.5), otros dentarios poseen dientes más gráciles y altos, con coronas menos expandidas (Figura 1.6). Esta distinción no es muy clara en la mayoría de los casos debido a la mala preservación de los especímenes y podría corresponder a una variación intraespecífica o interespecífica, que por el momento no puede ser resuelta.

\section{Consideraciones finales}

El género Liolaemus incluye más de dos centenares de especies y anualmente, se describen varias especies nuevas del género. Se distribuye en los Andes desde Perú y Bolivia hasta Tierra del Fuego, y desde la costa del Pacífico hasta las del Atlántico en Argentina, Uruguay y Brasil (Donoso-Barros 1966; Cei 1986, 1993). Es uno de los géneros de lagartos con la distribución latitudinal, altitudinal y climática más amplia, incluyendo especies que habitan por sobre los 5.000 metros sobre el nivel del mar (Cei 1986, 1993). Muchas especies son de amplia distribución geográfica y ambiental mientras que otras son endémicas, con distribuciones muy restringidas y poblaciones poco conocidas, algunas amenazadas de extinción (Scolaro 2005; Breitman et al. 2014).

Los fósiles más antiguos de Liolaemus son del Mioceno temprano de Patagonia (Albino 2008), pero no vuelven a documentarse hasta el Cuaternario (Albino 2005). El hallazgo en OB1 es el segundo reconocimiento de Liolaemus en un sitio arqueológico de Argentina, que se agrega al del sitio Bi Aike Cueva 3, también de la provincia de Santa Cruz (Albino y Franco 2011) y al de Alero 12 de la provincia de Catamarca (Albino y Kligmann 2007). A pesar que en la actualidad el género se distribuye muy extensamente en toda la Patagonia (Cei 1986; Scolaro 2005) y que existen numerosos trabajos sobre microfauna procedente de sitios arqueológicos patagónicos, el hallazgo de Liolaemus en OB1 es un nuevo aporte donde se fundamenta explícitamente la asignación taxonómica y resulta el hallazgo más austral hasta el presente. El registro arqueológico sudamericano del género se completa con el reconocimiento en la cueva Baño Nuevo 1 de la región de Aysén, al sur de Chile (Nuñez et al. 2005) donde se recuperaron un total de 99 restos entre maxilares y dentarios correspondientes a un mínimo de 25 individuos. La particularidad del hallazgo de OB1 radica en que se trata de una cantidad y diversidad muy superior de elementos esqueletarios, incluyendo no sólo dentarios y maxilares, sino también otros huesos, que corresponderían a una cantidad de individuos mayor a 100. La abundancia de bolos de regurgitación de Strigiformes hallados en OB1 explicaría la forma de ingreso de los restos de lagartijas (L'Heureux, 2008), coincidiendo con la propuesta de Núñez et al.(2005) para el sitio chileno.

Comparado con otros sitios de Argentina donde se reportaron restos herpetológicos, las lagartijas de OB1 son un componente muy significativo dentro del conjunto zooarqueológico muestreado y se destaca por la homogeneidad taxonómica ya que el único taxón presente es el iguánido Liolaemus. En este caso, el sesgo taxonómico se explica por las características inhóspitas del ambiente donde está emplazado el sitio, que resulta inhabitable para la mayor parte de los lagartos.

Mar del Plata, 9 de septiembre 2016

\section{Agradecimientos}

Se agradece a Luis Borrero y Ramiro Barberena por facilitar el material de lagartijas de OB1 para su estudio. A Mariana Morando y María Florencia Breitman por el préstamo de especímenes de diversas especies de Liolaemus utilizado en la comparación del material arqueológico. PIP CONICET 112-201501-00065CO.

\section{Referencias bibliográficas}

Albino, A. M. 2005. A late Quaternary lizard assemblage from the Southern Pampean Region of Argentina. Journal of Vertebrate Paleontology 25: 185-191.

Albino, A. M. 2008. Lagartos iguanios del Colhuehuapense (Mioceno temprano) de Gaiman (provincia del Chubut, Argentina). Ameghiniana 45: 775-782.

Albino A. M., R. H. Albino. 2004. Los Reptiles de los sitios arqueológicos de la Argentina y la bibliografía para su estudio. Comunicaciones del Museo Provincial de Ciencias Naturales "Florentino Ameghino" (Nueva Serie) 9: 1-16.

Albino, A. M., D. Kligmann. 2007. An accumulation of bone remains of two Liolaemus species in a Holocene 
archaeological site of the Argentinian Puna. AmphibiaReptilia 28: 154-158.

Albino, A. M., D. Kligmann. 2009. Inusual hallazgo de anfisbénidos (Squamata, Amphisbaenidae) en un yacimiento arqueológico de Argentina. Revista Española de Herpetología 23: 99-106.

Albino, A. M., N. Franco. 2011. Lagartijas (Iguania: Familia Liolaemidae) procedentes del sitio arqueológico Bi Aike Cueva 3 (Provincia de Santa Cruz, Argentina). Anales del Instituto de la Patagonia 39: 127-131.

Barberena, R., A. Blasi y C. Castiñeira. 2006. Geoarqueología en Pali Aike: Cueva Orejas de Burro 1 (Patagonia, Argentina). Magallania 34 119-138.

Breitman, M. F., I. Minoli, L. J. Avila, C. D. Medina, J. W. Sites y M. Morando. 2014. Lagartijas de la provincia de Santa Cruz, Argentina: distribución geográfica, diversidad genética y estado de conservación. Cuadernos de Herpetología 28: 83-110.

Cei, J. M. 1986. Reptiles del Centro, Centro-oeste y Sur de la Argentina. Herpetofauna de las Zonas Áridas y Semiáridas. Monografie 4, 1-527, Museo Regionale di Scienze Naturali, Torino.

Cei, J.M. 1993. Reptiles del Noroeste, Nordeste y Este de la Argentina. Herpetofauna de las Selvas Subtropicales, Puna y Pampas. Monografie 14, 1-949, Museo Regionale di Scienze Naturali. Torino.

Donoso-Barros, R. 1966. Reptiles de Chile, 1-458, Editorial Universidad de Chile. Santiago de Chile.

Etheridge, R. 1995. Redescription of Ctenoblepharys adspersa Tschudi, 1845, and the taxonomy of Liolaeminae (Reptilia: Squamata: Tropiduridae). American Museum Novitates 3142: 1-34.

Etheridge, R. 2000. A review of lizards of the Liolaemus wiegmannii group (Squamata, Iguania, Tropiduridae), and a history of morphological change in the sand-dwelling species.Herpetological Monographs 14: 293-352.

Frost, D. R., R. Etheridge. 1989. A phylogenetic analysis and taxonomy of iguanian lizards (Reptilia: Squamata). The University of Kansas, Museum of Natural History, Miscellaneous Publication 81: 1-65.

Frost, D. R., R. Etheridge, D. Janies y T. A. Titus. 2001. Total evidence, sequence alignment, evolution of polychrotid lizards, and a reclassification of the Iguania (Squamata: Iguania). American Museum Novitates 3343: 1-38.

Kligmann, D., A. M. Albino y E. Pais. 2010. ¿Anfisbenas para los dioses? Zooarqueología de una ofrenda animal. Izeta, A. D., G. L. Mengoni Goñalons (Eds.), De la Puna a las Sierras: Avances y Perspectivas en Zooarqueología Andina, 89-120, South America Archaeology Series, British Archaeological Reports.

L'Heureux, G. L. 2008.La arqueofauna del campo volcánico Pali Aike. El sitio Orejas de Burro 1, Santa Cruz, Argentina. Magallania 36: 65-78.

L'Heureux, G. L., Barberena, R. 2008. Evidencias bioarqueológicas en Patagonia meridional: el sitio Orejas de Burro 1 (Pali Aike, provincia de Santa Cruz). Intersecciones en Antropología 9: 11-24.

Lobo, F. 2001. A phylogenetic analysis of lizards of the Liolaemus chiliensis group (Iguania: Tropiduridae). Herpetological Journal 11: 137-150.

Núñez, H., T. W. Stafford y D. Frassinetti. 2005. Primer registro de fósiles de Liolaemus en Chile (Reptilia, Sauria). Noticiario mensual del Museo Nacional de Historia Natural 356: 3-7.

Quintana, C., D. Mazzanti. 2001. Selección y aprovechamiento de recursos faunísticos. D. Mazzanti y Quintana, C. (Eds.), Cueva Tixi: Cazadores y recolectores de las sierras de Tandilia Oriental. 1. Geología, Paleontología y Zooarqueología, Publicación Especial 1, 181-210, Laboratorio de Arqueología, Universidad Nacional de Mar del Plata, Mar del Plata.

Schulte, J. A., J. P. Valladares y A. Larson. 2003. Phylogenetic relationships within Iguanidae inferred using molecular and morphological data and a phylogenetic taxonomy of iguanian lizards. Herpetologica 59: 399419.

Scolaro, A. 2005. Reptiles Patagónicos: Sur. Una Guía de Campo, 1-80, Ediciones Universidad Nacional de la Patagonia, Trelew.

Van Devender, T. 1977. Observations on the argentine iguanid lizard Leiosaurus belli Duméril and Bibron (Reptilia, Lacertilia, Iguanidae). Journal of Herpetology 11: 238-241. 